duration of sick leave. Currently, oseltamivir prophylaxis is aimed at preventing influenza infection in unvaccinated or high-risk populations or to prevent secondary transmission in long-term care facilities in the context of an outbreak. ${ }^{4,5}$ We could not demonstrate that expanding oseltamivir PEP to all exposed HCWs was associated with a decrease in absenteeism, but this is a single-center experience in a setting with high HCW vaccination rates. However, both 2016-2017 and 2017-2018 influenza seasons were similar in Iowa, with a predominance of A (H3N2) influenza viruses and an overall vaccine effectiveness of only $40 \%{ }^{6-8}$ Absenteeism rates may have been similar due to HCWs presenting to work regardless of symptoms (ie, presenteeism). Expanded PEP may have been effective in those who took oseltamivir, but we could not analyze absenteeism stratified by PEP uptake. Expanding oseltamivir prophylaxis indications needs to be carefully assessed because it could contribute to the development of oseltamivir-resistant strains. ${ }^{9}$

In conclusion, we were unable to demonstrate reduced absenteeism by providing oseltamivir PEP to all exposed HCWs regardless of their vaccination status. Larger prospective studies may clarify the role of expanded PEP, especially during seasons with low vaccination effectiveness. Other preparedness strategies might be needed to achieve the right balance of minimizing sick leave by preventing HCW influenza acquisition while also minimizing presenteeism.

Acknowledgments. None.

Financial support. No financial support was provided for this analysis.
Conflicts of interest. All authors report no conflicts of interest relevant to this article.

\section{References}

1. Rule AM, Apau O, Ahrenholz SH, et al. Healthcare personnel exposure in an emergency department during influenza season. PLoS One 2018;13: e0203223.

2. Saxen H, Virtanen M. Randomized, placebo-controlled double blind study on the efficacy of influenza immunization on absenteeism of health care workers. Pediatr Infect Dis J 1999;18:779-783.

3. Recommended vaccines for healthcare workers. Centers for Disease Control and Prevention website. https://www.cdc.gov/vaccines/adults/recvac/hcw.html. Accessed October 16, 2018.

4. Influenza antiviral medications: summary for clinicians. Centers for Disease Control and Prevention website. https://www.cdc.gov/flu/professionals/ antivirals/summary-clinicians.htm. Accessed October 16, 2018.

5. Booy R, Lindley RI, Dwyer DE, et al. Treating and preventing influenza in aged care facilities: a cluster randomised controlled trial. PLoS One 2012;7:e46509.

6. Seasonal influenza vaccine effectiveness, 2004-2018. Centers for Disease Control and Prevention website. https://www.cdc.gov/flu/professionals/ vaccination/effectiveness-studies.htm. Accessed October 16, 2018.

7. Summary of the 2017-2018 influenza season. Centers for Disease Control and Prevention website. https://www.cdc.gov/flu/about/season/flu-season2017-2018.htm. Accessed October 16, 2018.

8. Summary of the 2016-2017 influenza season. Centers for Disease Control and Prevention website. https://www.cdc.gov/flu/about/season/flu-season2016-2017.htm. Accessed October 16, 2018.

9. Graitcer SB, Gubareva L, Kamimoto L, et al. Characteristics of patients with oseltamivir-resistant pandemic (H1N1) 2009, United States. Emerg Infect Dis 2011;17:255-257.

\title{
Establishing a mass prophylaxis clinic during a hospital scabies outbreak
}

\author{
Caitlin M. Adams Barker MSN, RN, $\mathrm{CIC}^{1}$, M. James Alexander $\mathrm{BS}^{2}$ and Antonia L. Altomare DO, MPH ${ }^{1,3}$ \\ ${ }^{1}$ Collaborative Healthcare-Associated Infection Prevention Program, Dartmouth-Hitchcock Medical Center, Lebanon, New Hampshire, ${ }^{2}$ Emergency Management \\ Program, Dartmouth-Hitchcock Medical Center, Lebanon, New Hampshire and ${ }^{3}$ Section of Infectious Disease and International Health, Dartmouth-Hitchcock \\ Medical Center, Lebanon, NH, US
}

To the Editor-An immunocompromised hospital inpatient with Norwegian (crusted) scabies went undiagnosed for 6 weeks without isolation or treatment. A literature review revealed little guidance on the logistics of providing mass prophylaxis to exposed individuals. We used a hospital-based incident command system to establish a mass prophylaxis clinic.

A patient with a history of gynecological malignancy undergoing chemotherapy was admitted to a 33-bed hematology-oncology, bone marrow transplant unit at our academic medical center in northern New England. The patient had skin

Author for correspondence: Caitlin M. Adams Barker MSN, RN, CIC, 1 Medical Center Drive, Lebanon, NH 03756. E-mail: Caitlin.m.adams.barker@hitchcock.org PREVIOUS PRESENTATION: These data were previously reported as a poster presentation at the 2015 SHEA Annual Conference on May 15, 2015, in Orlando, Florida.

Cite this article: Adams Barker CM, et al. (2019). Establishing a mass prophylaxis clinic during a hospital scabies outbreak. Infection Control \& Hospital Epidemiology 2019, 40, 261-263. doi: 10.1017/ice.2018.322 plaques and rash of unknown etiology, which went undiagnosed for 43 days due to the absence of pain or itching. Dermatology was consulted, and an exam revealed diffuse crusted hyperkeratotic scaly macules and papules including plaques in the webbing of the fingers. Skin scraping with mineral oil preparation revealed at least 5 scabies mites. The patient was diagnosed with crusted scabies, placed on contact precautions, and treated with oral ivermectin and topical permethrin.

Upon notification, the infection prevention (IP) program reviewed guidance from the Centers for Disease Control and Prevention and additional literature to assess recommendations for providing prophylaxis in the setting of a prolonged period of undiagnosed crusted scabies in a healthcare facility. Modified institution-wide prophylaxis for patients, staff, visitors, and household contacts was deemed necessary to prevent an outbreak of scabies within the facility. The literature lacked guidance on 


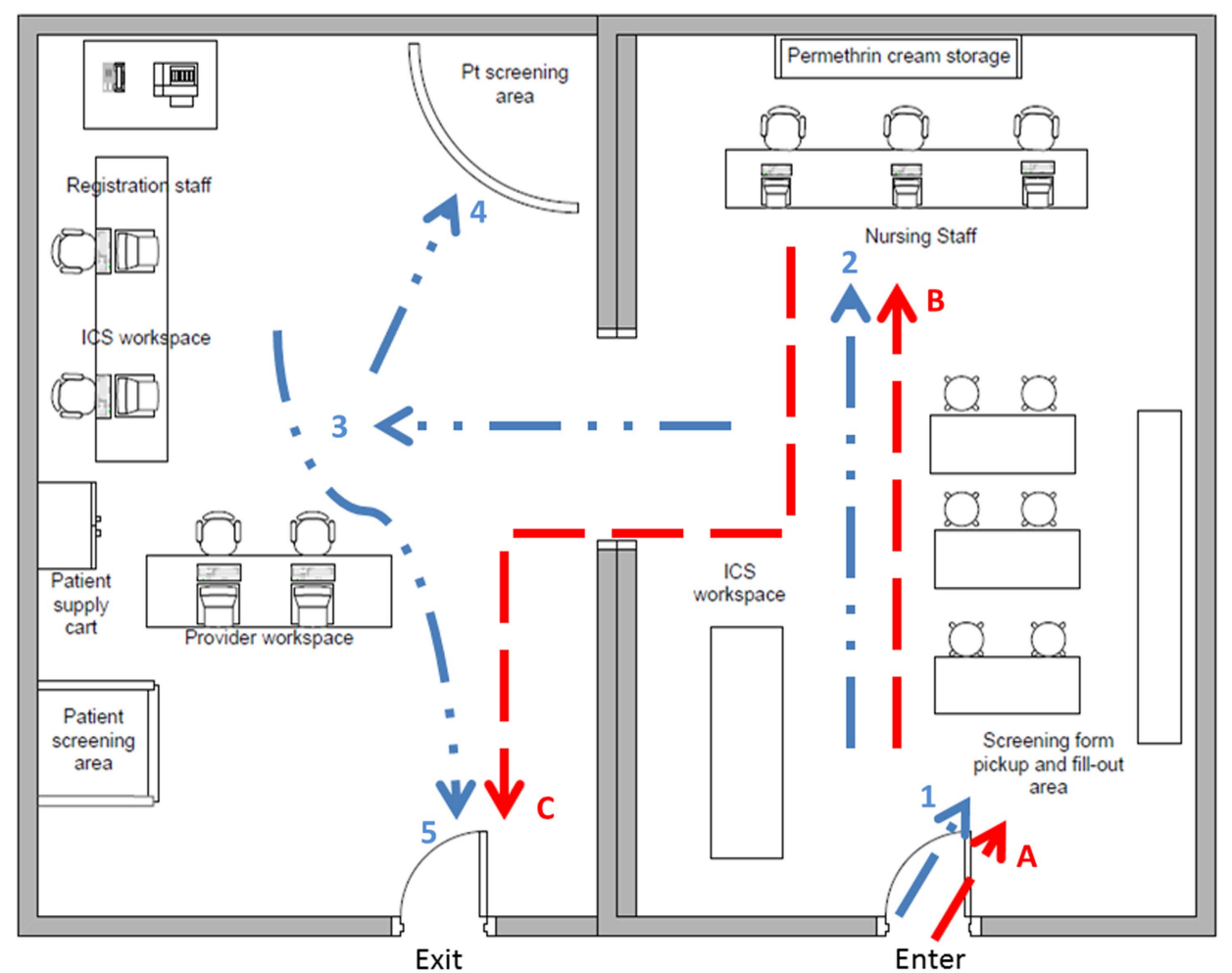

Fig. 1. Mass prophylaxis clinic set up. The dashed line (A-C) presents patient flow of a patient not needing provider examination. The dotted line (1-5) represents patient flow of a patient needing a provider examination.

operationalization of a large-scale intervention. The hospital incident command system was activated to assist in establishing methods for notifying staff, patients, and visitors of their exposure and to manage the logistics of providing prophylaxis to a large number of people over a short period of time.

The hospital incident command system is activated in situations in which a large number of people may be affected. Past activations included network outages and contamination of the city water supply. Notifications are sent via a paging system and by telephone to members of the incident command team alerting them of the activation. A dedicated conference room is used as a meeting space and a dedicated phone line is established for conference calling. Those responding to the activation include hospital administrators, subject-matter experts, public information officers, and emergency management (EM) staff. Because this incident involved patients, staff, and visitors in inpatient and ancillary areas, more team members were utilized than in a typical activation.

The incident command team met 3 times daily, and smaller subcommittees met throughout the day to discuss physical setup, location, staffing and dates of the clinic, acquisition of medication, and communication with those exposed. A walk-in clinic was deemed the most efficient way to provide prophylaxis to those exposed. Based on availability and ease of dosing, topical permethrin was selected as prophylaxis.

Staff who had the closest contact with the index patient, as determined by a review of the medical record, were notified by email of their exposure and the need to acquire prophylaxis for themselves and their household members. Dates, times, and locations of the clinics were included in this communication. A review of the affected unit's census was performed to identify patients who were admitted to the affected unit during the specified period, and a letter was sent to those discharged notifying them of their possible exposure and providing information for attending the clinics. For those who had passed away since their admission, families were notified by a phone call from the nursing director and prophylaxis was offered to the household members. Patients currently on the affected unit at the time of the outbreak were notified by their attending physician and were prescribed permethrin or ivermectin as prophylaxis. A public announcement was made in the local newspaper, television, and radio stations to alert members of the public who may have visited the affected unit during the exposure period. An information hotline was set up through the department of health for anyone with questions about scabies or the clinics. The department of health, in collaboration with the IP program, released a Health Alert Network (HAN) communication regarding the exposure, and a website was created with contact information and frequently asked questions.

Two adjoining conference rooms were used as clinic space. Figure 1 illustrates the set up and flow of the clinics. Everyone who presented to the clinic was screened using a paper screening tool. All who indicated being on the affected unit during the exposure period or who were a household member of someone who was, were offered prophylaxis. A cohort order was written by the medical director of EM, and a nurse protocol was used to place the order for those needing prophylaxis. The order allowed for heads of households to obtain medication for those living with them. All patients were assessed for allergies or contraindications to permethrin cream before it was given, and heads of household were responsible for reporting allergies and contraindications of those who were not present. 
Adjacent to the screening, a prescribing and dispensing area was established for patient examination. Privacy screens were used to create examination areas, and personal protective equipment, patient gowns, and linens were provided. Physicians staffed the clinic to assess patients who were concerned that they might have scabies. Supplies for skin scrapings and slide preparation were available, and a lab technician was on-call to collect specimens for processing.

More than 1,000 doses of permethrin were distributed during the clinics. There was 1 confirmed case of scabies and 3 probable cases of secondary transmission as a result of this outbreak.
Establishing a centrally located clinic using the incident command system structure provided rapid and effective screening and prophylactic treatment of scabies to prevent a larger outbreak of scabies within the institution.

Acknowledgments. None.

Financial support. No financial support was provided relevant to this article.

Conflicts of interest. All authors report no conflicts of interest relevant to this article.

\title{
Low prevalence of the $\mathrm{mcr}-1$ gene among carbapenemase- producing clinical isolates of Enterobacterales
}

\author{
Tanise Vendruscolo Dalmolin ${ }^{1,2}$, Priscila Lamb Wink ${ }^{1}$, Daiana de Lima-Morales ${ }^{1}$ and Afonso Luís Barth ${ }^{1,2}$ \\ ${ }^{1}$ Laboratório de Pesquisa em Resistência Bacteriana, LABRESIS, Centro de Pesquisa Experimental, Hospital de Clínicas de Porto Alegre, Porto Alegre, RS, Brazil \\ and ${ }^{2}$ Programa de Pós-Graduação em Ciências Farmacêuticas, Faculdade de Farmácia, Universidade Federal do Rio Grande do Sul, Porto Alegre, RS, Brazil
}

To the Editor-Polymyxins are the last resort for the treatment of infections caused by multidrug-resistant bacteria, in particular carbapenem-resistant Enterobacterales (CRE). Resistance to polymyxins used to be due only to chromosomal mutations, but in November 2015, Liu et $\mathrm{al}^{1}$ described for the first time a colistin resistance mechanism mediated by a new gene $(\mathrm{mcr}-1)$ that was present in a transferable plasmid. The $m c r-1$ has already been described on most continents, being detected in different species and obtained from several sources, including carbapenemaseproducing clinical isolates. ${ }^{2,3}$ Infections due to clinical isolates harboring the $m c r-1$ and a carbapenem resistance gene is of particular concern because the treatment options would be seriously compromised. ${ }^{4}$

The aim of this study was to evaluate the prevalence of carbapenemase and $\mathrm{mcr}$ - 1 genes co-occurring among Enterobacterales clinical isolates in southern Brazil between April 2013 and May 2018.

We evaluated the occurrence of the $m c r-1$ gene among 4,778 isolates of Enterobacterales with reduced susceptibility to carbapenems obtained from an epidemiologic study in several hospitals in southern Brazil. All isolates were submitted to multiplex realtime polymerase chain reaction with high-resolution melting (RT-PCR-HRM) analysis with primers for $b l a_{\mathrm{KPC}}, b l a_{\mathrm{NDM}}$, $b l a_{\mathrm{OXA}-48 \text {-like, }} b l a_{\mathrm{GES}}, b l a_{\mathrm{IMP}}$, and $b l a_{\mathrm{VIM}}$ and presented positive results for at least 1 of the carbapenemase gene(s) tested.

The presence of the $m c r-1$ gene was evaluated by pooling 10 isolates together and submitting them to DNA extraction and conventional PCR with specific primers for the $m c r-1$ gene. ${ }^{1}$ All isolates from a pool with $m c r-1$ positive result were retested individually by the same conventional PCR to identify the isolate (s) that presented the gene. The amplicons from the individual

Author for correspondence: Afonso Luís Barth, LABRESIS, Hospital de Clínicas de Porto Alegre, Ramiro Barcelos 2350, Porto Alegre, Brazil. E-mail: albarth@hcpa.edu.br Cite this article: Dalmolin TV, et al. (2019). Low prevalence of the $\mathrm{mcr}$ - 1 gene among carbapenemase-producing clinical isolates of Enterobacterales. Infection Control \& Hospital Epidemiology 2019, 40, 263-264. doi: 10.1017/ice.2018.301 isolates with positive result in the conventional PCR were submitted to Sanger sequencing and were confirmed as the $m c r-1$ variant. The minimal inhibitory concentrations (MICs) of several antibiotics were evaluated using broth microdilution method for the individual isolates positive for the $m c r-1$ gene, and the results were interpreted according to European Committee on Antimicrobial Susceptibility Testing (EUCAST) guidelines. ${ }^{5}$

We found only 5 isolates that presented the $m c r-1$ gene and a carbapenemase gene. All coharboring isolates presented the $\mathrm{mcr}-1$ and the $b l a_{\mathrm{KPC}}$ genes. We obtained 2 coharboring isolates (Klebsiella pneumoniae $3111 \mathrm{~F}$ and Escherichia coli 3431F) in 2014, 1 coharboring isolate (E. coli 5798F) in 2016, and the other 2 coharboring isolates (K. pneumoniae $6701 \mathrm{~F}$ and E. coli $6699 \mathrm{~F}$ ) in 2018. All 5 isolates were recovered from rectal swabs, with exception of $E$. coli 6699 F, which was recovered from an ascites fluid.

Moreover, 4 isolates presented low-level resistance to colistin $(4 \mathrm{mg} / \mathrm{L})$, and 1 isolate (K. pneumoniae $6701 \mathrm{~F})$ was susceptible to colistin $(0.25 \mathrm{mg} / \mathrm{L})$. All isolates were resistant to ertapenem, meropenem, imipenem, and ciprofloxacin and were susceptible to tigecycline. Susceptibility to aminoglycosides was variable, with most isolates susceptible to gentamicin and intermediate to amikacin (Table 1).

The prevalence of the $m c r-1$ gene was very low $(0.1 \%)$ among carbapenemase-producing Enterobacterales (CPE) in our study. This rate is lower than that reported in Portugal, where $6.69 \%$ of the $\mathrm{CPE}$ isolates from colonized and infected patients were positive for the $m c r-1$ gene. $^{6}$ In Belgium, the prevalence reported was $<1 \%$ among CRE of human origin. ${ }^{7}$ These findings demonstrate that the prevalence of $m c r-1$ with carbapenemase genes is normally very low, although it can differ among countries.

The $m c r-1$ gene is usually evaluated only among colistinresistant isolates (MIC $>2 \mathrm{mg} / \mathrm{L}$ ); however, the isolate $K$. pneumoniae $6701 \mathrm{~F}$ was susceptible to colistin. Some isolates are mor-1 positive; nonetheless they are colistin susceptible. One explanation for this is the assumption that the gene might be truncated in isolates positive for the $m c r-1$ but susceptible to 